\title{
Fixed points and Stability of Damped and Unforced Duffing Equation, Solution and Graphs for Displacement and Velocity of that Equation using Matlab
}

\author{
Ajaya Kumar Singh
}

Department of Mathematics, Ekamra College, Bhubaneswar, Odisha, India

\section{ABSTRACT}

The object of the present paper is to find the fixed points and their stability by matrix method of the duffing equation (damped $(\delta \neq 0)$ and unforced / undriven

$(\gamma=0)) \ddot{x}+\delta \dot{x}+\left(v x^{3} \pm \rho x\right)=\gamma \cos (\omega t)$,

$x=x(t), t=$ time period

using special case $v=1=\rho$ and taking the minus sign in (1), it becomes $\ddot{x}+\delta \dot{x}+\left(x^{3}-x\right)=0$

The duffing equation can be expressed in $y_{2}(t)$ and $\dot{y}_{2}(t)$ shown by MATLAB program and their graphs.

Keywords : Duffing Equation, Jacobi Elliptic Functions, Period, Boundedness

\section{INTRODUCTION}

The concept of Duffing equation or Duffing Oscillator was named after Georg Duffing (1861 - 1944), is a non -linear second order differential equation used to model certain damped $(\delta \neq 0)$ and driven $(\gamma \neq 0)$ oscillators.

The equation is given by

$\ddot{x}+\delta \dot{x}+\left(\rho x \pm v x^{3}\right)=\gamma \cos (\omega t)$

(1)

where $x(t)=$ displacement at time $t$

$$
\dot{x}=\frac{d}{d t}(x(t))=\text { velocity }
$$

and $\ddot{x}=\frac{d^{2}}{d t^{2}}(x(t))=$ acceleration.

The numbers $\delta, \rho, \nu, \gamma, \omega$ are given constants.

\section{Parameters}

The parameters of equation (1) are

- $\delta$ controls the amount of damping

- $\rho$ controls the linear stiffness

- $v$ controls the amount of non-linearity

- $\gamma$ is the amplitude of the periodic driving force

- $\omega$ is the angular frequency of the periodic driving force
Note

- If $v=0$, then (1) describes the damped and

- driven simple harmonic oscillator.

- If $\gamma=0$, then (1) describes the undamped

- oscillator.

- The restoring force provided by the non-linear

spring is $\rho x+v x^{3}$

Case - I

If $\rho>0$ and $v>0$, then (2) is called a hardening spring

Case - II

If $\rho>0$ and $v<0$, then (2) is called a softening spring

The number of parameters in (1) can be reduced to two through scaling by the excursion $x$ and the time t. Now, let $\tau=t \sqrt{\rho}$ and $y=\frac{x \rho}{\gamma}, \rho>0 \quad$ and substituting it in (1), we get $\ddot{y}+2 \eta \dot{y}+y+\epsilon y^{3}=$ $\cos (\omega \tau)$

where $\eta=\frac{\delta}{2 \sqrt{\rho}}, \epsilon=\frac{v \gamma^{2}}{\rho^{2}}, \sigma=\frac{\omega}{\sqrt{\rho}}$

and the dots denote differentiation of $y(\tau)$ with respect

to $\tau$. 
This shows that the solutions to (1) can be described in terms of the three parameters $\eta, \epsilon, \sigma$ with two initial conditions $y\left(t_{0}\right)$ and $\dot{y}\left(t_{0}\right)$.

\section{Equilibrium Points}

The equilibrium points stable and unstable are at (2)

\section{Case - I}

If $\rho>0$, then the equilibrium point is at $x=0$.

\section{Case - II}

If $\rho<0$ and $>0$, then the equilibrium points are at $x= \pm \sqrt{-\frac{\rho}{v}}$

\section{Methods of Solution}

Many approximate solutions for the duffing equation are

- By Fourier series method.

- By Frobenius method which yields a complex solution.

- By numeric methods such as Euler's method and Runge-Kutta.

- By Homotopy analysis method which yields approximate solutions of the duffing equation.

- In the special case of the undamped $(\delta=0)$ and undriven $(\gamma=0)$ duffing equation an exact solution can be obtained using Jacobi's elliptic functions.

\section{DAMPED AND UNFORCED DUFFING EQUATION BY MATRIX METHOD}

The duffing equation is

$\ddot{x}+\delta \dot{x}+\left(v x^{3} \pm \rho x\right)=\gamma \cos (\omega t)$,

$x=x(t), t=$ time perio

In this chapter, my aim is to find the fixed points and their stability by matrix method of the duffing equation (damped $(\delta \neq 0)$ and unforced / undriven $(\gamma=0))$ using special case $v=1=\rho$ and taking the minus sign in (1) becomes

$$
\ddot{x}+\delta \dot{x}+\left(x^{3}-x\right)=0
$$

Now, (2) transforms to a system of first order differential equations. (See [4], [10])

Setting

$$
\begin{aligned}
& \dot{x}=y \\
& \dot{y}=x-x^{3}-\delta y
\end{aligned}
$$

Now, for the fixed point, the system of differential equations are

$$
\dot{x}=y=0
$$

and $\dot{y}=x-x^{3}-\delta y$

$\Rightarrow 0=x\left(1-x^{2}\right)-0$

$\Rightarrow 0=x\left(1-x^{2}\right)$

$\Rightarrow x\left(1-x^{2}\right)=0$

$\Rightarrow x=0 \quad$ or $\quad\left(1-x^{2}\right)=0$

$\Rightarrow x=0$ or $x^{2}=1$

$\Rightarrow x=0 \quad$ or $\quad x= \pm 1$

$\therefore$ The fixed points are $(-1,0),(1,0)$ and $(0,0)$.

Analysis of the stability of the fixed points by matrix method to be determined.

Differentiating (3) and (4) gives

$\ddot{x}=\dot{y}$

$$
=x-x^{3}-\delta y
$$

$\ddot{y}=\left(1-3 x^{2}\right) \dot{x}-\delta \dot{y}$

Which can be written as the matrix equation

$$
\left[\begin{array}{c}
\ddot{x} \\
\ddot{y}
\end{array}\right]=\left[\begin{array}{cc}
0 & 1 \\
\left(1-3 x^{2}\right) & -\delta
\end{array}\right]\left[\begin{array}{c}
\dot{x} \\
\dot{y}
\end{array}\right]
$$

Case - I (for $(0,0))$

The characteristic equation is

$$
\operatorname{det}(A-\lambda I)=0,
$$

$\lambda=$ characteristic values which to be determined

$$
\begin{aligned}
& \operatorname{det}\left(\left[\begin{array}{cc}
0 & 1 \\
(1-0) & -\delta
\end{array}\right]-\left[\begin{array}{ll}
\lambda & 0 \\
0 & \lambda
\end{array}\right]\right)=0 \\
& \Rightarrow \operatorname{det}\left(\left[\begin{array}{cc}
0-\lambda & 1-0 \\
1-0 & -\delta-\lambda
\end{array}\right]\right)=0 \\
& \Rightarrow \operatorname{det}\left(\left[\begin{array}{cc}
-\lambda & 1 \\
1 & -\delta-\lambda
\end{array}\right]\right)=0 \\
& \Rightarrow-\lambda(-\delta-\lambda)-1=0
\end{aligned}
$$


Since, $\sqrt{\delta^{2}+4}>|\delta|$

$\lambda$ has one positive root only.

So, this fixed point $(0,0)$ is unstable.

Case II (for $( \pm 1,0))$

The characteristic equation is

$$
\begin{aligned}
& \operatorname{det}\left(\left[\begin{array}{cc}
0 & 1 \\
(1-3) & -\delta
\end{array}\right]-\left[\begin{array}{ll}
\lambda & 0 \\
0 & \lambda
\end{array}\right]\right)=0 \\
& \Rightarrow \operatorname{det}\left(\left[\begin{array}{cc}
0-\lambda & 1-0 \\
-2-0 & -\delta-\lambda
\end{array}\right]\right)=0 \\
& \Rightarrow \operatorname{det}\left(\left[\begin{array}{cc}
-\lambda & 1 \\
-2 & -\delta-\lambda
\end{array}\right]\right)=0 \\
& \Rightarrow-\lambda(-\delta-\lambda)+2=0 \\
& \Rightarrow \lambda^{2}+\lambda \delta+2=0 \\
& \Rightarrow \lambda=\frac{\left(-\delta \pm \sqrt{\delta^{2}-4 \times 2 \times 1}\right)}{2 \times 1} \\
& \Rightarrow \lambda_{ \pm 1,0}=\frac{1}{2}\left(-\delta \pm \sqrt{\delta^{2}-8}\right)
\end{aligned}
$$

For $\delta>0$

So, $\lambda_{ \pm 1,0}$ is imaginary.

$\lambda$ has complex roots.

So, the point $( \pm 1,0)$ is asymptotically stable.

Case III (for Undamped $(\delta=0)$ )

$$
\begin{aligned}
\lambda_{ \pm 1,0} & =\frac{1}{2}\left(-\delta \pm \sqrt{\delta^{2}-8}\right) \\
\Rightarrow \lambda_{ \pm 1,0} & =\frac{1}{2}(0 \pm \sqrt{0-8}) \\
\Rightarrow \lambda_{ \pm 1,0} & =\frac{1}{2}(0 \pm \sqrt{-8}) \\
\Rightarrow \lambda_{ \pm 1,0} & =\frac{1}{2}(0 \pm i 2 \sqrt{2}) \\
\Rightarrow \lambda_{ \pm 1,0} & = \pm i \sqrt{2}
\end{aligned}
$$

Since, $\delta=0$

So, the fixed point $\lambda_{ \pm 1,0}$ has an imaginary root and is linearly stable. See $[10]$

Case IV for $(\delta \in(-2 \sqrt{2}, 0))$

The fixed point $\lambda_{ \pm 1,0}$ has a real root and is unstable.

Case $\mathrm{V}$ for $(\delta=-2 \sqrt{2})$

$\lambda_{ \pm 1,0}=\sqrt{2}$

The fixed point $\lambda_{ \pm 1,0}$ has a positive real root and is unstable.

Case VI for $(\delta<-2 \sqrt{2})$

The fixed points $\lambda_{ \pm 1,0}$ are positive real roots and are unstable.

\section{SOLUTIONS AND GRAPHS OF ISPLACEMENT AND VELOCITY FOR DUFFING EQUATION USING MATLAB}

Consider the duffing equation

$$
\ddot{x}+\delta \dot{x}+\left(v x^{3} \pm \rho x\right)=\gamma \cos (\omega t)
$$

where $x=x(t), t=$ time period

Substituting $y_{1}(t)=x(t)$ and $y_{2}(t)=\dot{x}(t)$ and plus sign in (1), we get

$$
\begin{aligned}
\dot{y}_{1}(t) & =\dot{x}(t)=y_{2}(t) \\
\dot{y}_{2}(t) & =\ddot{x}(t) \\
& =-\delta y_{2}(t)-\rho y_{1}(t)-v y_{1}^{3}(t)+\gamma \cos (\omega t)
\end{aligned}
$$

Consider the parameters $\rho=1, v=-1, \gamma=3$, $\delta=2, \omega=1$.

In this chapter, the duffing equation can be expressed in $y_{2}(t)$ and $\dot{y}_{2}(t)$ shown by MATLAB program and their graphs. (see [4], [1], [10])

\section{Program :}

\section{M-file}

function dydt $=$ duffing_2 $(t, y)$

\%Local parameters

rho $=1$;

$\mathrm{nu}=-1$;

gamma $=3$;

delta $=2$;

omega $=1$;

\%State vector is $[\mathrm{y}(\mathrm{t}) ; \mathrm{ydot}(\mathrm{t})]$;

$\operatorname{dydt}(1)=y(2)$;

$\operatorname{dydt}(2)=-\operatorname{delta}^{*} \mathrm{y}(2)-\operatorname{rho}^{*}(\mathrm{y}(1))-$

$\mathrm{nu}^{*}\left(\mathrm{y}(1)^{\wedge} 3\right)+$ gamma $^{*} \cos \left(\right.$ omega $\left.^{*} \mathrm{t}\right)$;

dydt $=$ dydt'; $^{\prime}$

return

\section{Command Window :}

$>$ figure

$>>\mathrm{t}, \mathrm{y}]=$ ode45(@duffing_2, [0,5],

$$
[0 ; 0]) \text {; }
$$

$>\mathrm{p}=\operatorname{plot}\left(\mathrm{t}, \mathrm{y},{ }^{\prime * \prime}\right)$

$>$ title('DUFFING EQUATION $: \backslash$ rho $=1$, 
$\backslash \mathrm{nu}=-1, \backslash$ gamma $=3$,

\delta $=2$, lomega $=1^{\prime}$ )

>> xlabel('Time ( $\mathrm{t}$ ) in sec')

>> ylabel('Solution $y 2(t)$ and

Derivative of $\left.\mathrm{y} 2(\mathrm{t})^{\prime}\right)$

>> legend('y2(t)', 'Derivative of y2(t)')

\section{Result}

\begin{tabular}{|c|c|c|}
\hline 0 & 02953 & 25766 \\
\hline 0.0000 & 0.3686 & 2.6587 \\
\hline 0.0000 & 0.4419 & 2.7409 \\
\hline 0.0001 & 0.5152 & 2.8418 \\
\hline 0.0001 & 0.6129 & 2.9428 \\
\hline 0.0002 & 0.7106 & 3.0438 \\
\hline 0.0002 & 0.8083 & 3.1448 \\
\hline 0.0003 & 0.9059 & 3.2631 \\
\hline 0.0004 & 1.0001 & 3.3814 \\
\hline 0.0008 & 1.0943 & 3.4997 \\
\hline 0.0012 & 1.1885 & 3.6179 \\
\hline 0.0017 & 1.2827 & 3.7429 \\
\hline 0.0021 & 1.3813 & 3.8679 \\
\hline 0.0042 & 1.4799 & 3.9929 \\
\hline 0.0063 & 1.5785 & 4.1179 \\
\hline 0.0084 & 1.6771 & 4.2313 \\
\hline 0.0104 & 1.7690 & 4.3447 \\
\hline 0.0209 & 1.8609 & 4.4580 \\
\hline 0.0314 & 1.9528 & 4.5714 \\
\hline 0.0418 & 2.0447 & 4.6785 \\
\hline 0.0523 & 2.1367 & 4.7857 \\
\hline 0.0947 & 2.2286 & 4.8928 \\
\hline 0.1372 & 2.3205 & 5.0000 \\
\hline 0.1796 & 2.4124 & \\
\hline 0.2220 & 2.4945 & \\
\hline
\end{tabular}

\begin{tabular}{|c|c|c|c|}
\hline 0 & 0 & 0.9424 & 0.7023 \\
\hline 0.0000 & 0.0001 & 1.0084 & 0.6358 \\
\hline 0.0000 & 0.0001 & 1.0677 & 0.5666 \\
\hline 0.0000 & 0.0002 & 1.1201 & 0.4956 \\
\hline 0.0000 & 0.0002 & 1.1653 & 0.4228 \\
\hline 0.0000 & 0.0005 & 1.2010 & 0.3533 \\
\hline 0.0000 & 0.0007 & 1.2303 & 0.2818 \\
\hline 0.0000 & 0.0010 & 1.2528 & 0.2078 \\
\hline 0.0000 & 0.0012 & 1.2683 & 0.1308 \\
\hline 0.0000 & 0.0025 & 1.2767 & 0.0498 \\
\hline 0.0000 & 0.0037 & 1.2774 & -0.0357 \\
\hline 0.0000 & 0.0050 & 1.2700 & -0.1261 \\
\hline 0.0000 & 0.0062 & 1.2540 & -0.2220 \\
\hline 0.0000 & 0.0125 & 1.2321 & -0.3126 \\
\hline 0.0001 & 0.0187 & 1.2026 & -0.4076 \\
\hline 0.0001 & 0.0249 & 1.1650 & -0.5066 \\
\hline 0.0002 & 0.0310 & 1.1192 & -0.6088 \\
\hline 0.0006 & 0.0614 & 1.0513 & -0.7376 \\
\hline 0.0014 & 0.0912 & 0.9704 & -0.8665 \\
\hline 0.0026 & 0.1204 & 0.8765 & -0.9915 \\
\hline 0.0040 & 0.1489 & 0.7703 & -1.1081 \\
\hline 0.0126 & 0.2581 & 0.6320 & -1.2273 \\
\hline 0.0257 & 0.3575 & 0.4811 & -1.3194 \\
\hline 0.0428 & 0.4476 & 0.3212 & -1.3780 \\
\hline 0.0636 & 0.5286 & 0.1565 & -1.3995 \\
\hline 0.1068 & 0.6484 & -0.0176 & -1.3817 \\
\hline 0.1580 & 0.7444 & -0.1870 & -1.3256 \\
\hline 0.2154 & 0.8184 & -0.3475 & -1.2371 \\
\hline 0.2775 & 0.8724 & -0.4957 & -1.1260 \\
\hline 0.3651 & 0.9169 & -0.6170 & -1.0143 \\
\hline 0.4556 & 0.9337 & -0.7252 & -0.8962 \\
\hline 0.5467 & 0.9272 & -0.8200 & -0.7755 \\
\hline 0.6363 & 0.9021 & -0.9012 & -0.6548 \\
\hline 0.7196 & 0.8649 & -0.9654 & -0.5419 \\
\hline 0.7988 & 0.8175 & -1.0175 & -0.4298 \\
\hline \multirow[t]{2}{*}{0.8733} & 0.7624 & -1.0575 & -0.3179 \\
\hline & & -1.0854 & -0.2048 \\
\hline
\end{tabular}


$\mathrm{p}=$

174.0133

175.0128

\section{Graph :}

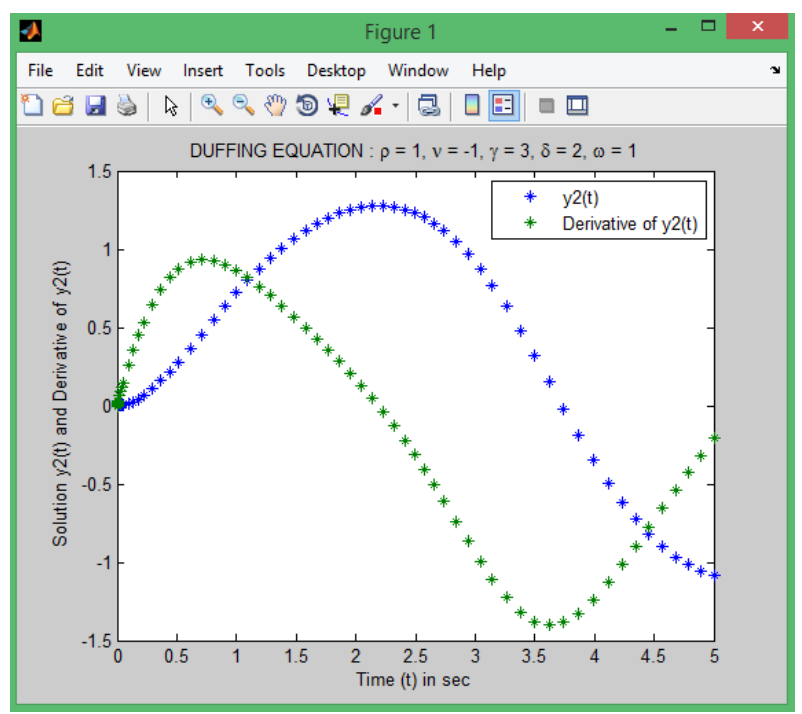

\section{REFERENCES}

[1]. A. K. Singh, Matlab Programming with Practical, Kalyani Publishers, Ludhiana, (2017).

[2]. I. Kovacic and M. J. Brennan, The Duffing Equation: Non Linear Oscillators and their Behaviour, Willey, (2011).

[3]. S. V. Wiggins, Application to the Dynamics of the Damped, Forced Duffing Oscillator. An Introduction to Applied Nonlinear Dynamical Systems and Chaos. Spring-Verlag, New York.

[4]. J. S. Roy and S. Padhy, A Course on Ordinary and Partial Differential Equations (with Applications), Kalyani Publishers, Ludhiana, (2014).

[5]. P. F. Bird and M. D. Friedman, Handbook of elliptic integrals for Engineers and Scientists, Springer, (1971).

[6]. E. Ott, Chaos in Dynamical Systems, Cambridge University Press, New York, (1993).
[7]. A. H. Salas and E. Jairo H. Castillo Applied Mathematical Sciences, 8(176), (2014).

[8]. J. V. Armitage and W. F. Eberlein, Eliptic Functions, Cambridge University Press, (2006).

[9]. C. M. Bender and S. A. Orszag, Advanced Mathematical Methods for Scientists and Engineers, McGraw - Hill, New York, (1978).

[10]. D. Zwillinger, Handbook of Differential Equations, Academic Press, Boston, (1997).

[11]. A. N. Nayfeth and D.T. Mook, Non-linear Oscillations, John Wiley, New York, (1973).

\section{Cite this article as :}

Ajaya Kumar Singh, "Fixed points and Stability of Damped and Unforced Duffing Equation, Solution and Graphs for Displacement and Velocity of that Equation using Matlab", International Journal of Scientific Research in Science and Technology (IJSRST), Online ISSN : 2395-602X, Print ISSN : 23956011, Volume 7 Issue 1, pp. 35-39, January-February 2020. Available at doi : https://doi.org/10.32628/IJSRST20715

Journal URL : http://ijsrst.com/IJSRST20715 